\title{
A new look at energy harvesting half-duplex DF power splitting protocol relay network over rician channel in case of maximizing capacity
}

\author{
Phu Tran Tin, Minh Tran², Tan N. Nguyen ${ }^{3}$, Thanh-Long Nguyen ${ }^{4}$ \\ ${ }^{1}$ Faculty of Electronics Technology, Industrial University of Ho Chi Minh City, Ho Chi Minh City, Vietnam \\ ${ }^{2}$ Optoelectronics Research Group, Faculty of Electrical and Electronics Engineering, \\ Ton Duc Thang University, Ho Chi Minh City, Vietnam \\ ${ }^{3}$ Wireless Communications Research Group, Faculty of Electrical and Electronics Engineering, \\ Ton Duc Thang University, Ho Chi Minh City, Vietnam \\ ${ }^{4}$ Center for Information Technology, Ho Chi Minh City University of Food Industry, Ho Chi Minh City, Vietnam
}

\section{Article Info \\ Article history: \\ Received Aug 29, 2018 \\ Revised Nov 19, 2018 \\ Accepted Oct 1, 2018 \\ Keywords: \\ Decode-and-forward (DF) \\ Energy harvesting $(\mathrm{EH})$ \\ Ergodic capacity \\ Power splitting protocol \\ Relay network}

\begin{abstract}
In this paper, the system performance in term of the ergodic capacity of a half-duplex decode-and-forward relaying network over Rician Fading Channel is investigated. The power splitting protocol is proposed for the system model. For this purpose, the analytical mathematical expressions of the ergodic capacity in cases of maximize and no-maximize ergodic capacity are derived and discussed. Furthermore, the effect of various system parameters on the system performance is rigorously studied. Finally, the analytical results are also demonstrated by Monte-Carlo simulation in comparison with the analytical expressions. The research results show that the analytical mathematical and simulated results match for all possible parameter values for both schemes.
\end{abstract}

Copyright () 2019 Institute of Advanced Engineering and Science. All rights reserved.

\section{Corresponding Author:}

Tan N. Nguyen,

Wireless Communications Research Group,

Faculty of Electrical and Electronics Engineering,

Ton Duc Thang University,

Ho Chi Minh City, Vietnam.

Email: nguyennhattan@tdtu.edu.vn

\section{INTRODUCTION}

In the last decades, the proliferation of cellular networks and wireless sensor networks, have demanded the higher quality of power supply from the source node to wireless devices. In the conventional method, wireless devices are generally powered by batteries, which have to be replaced/recharged on time manually. In the communication network, the process of battery replacement can be linked to some disadvantages such as inconvenient, infeasible for some applications [1-4]. In the last decade, one of the best ways to transfer energy in the communication network is using RF signals. In the last time, the system performance of the energy harvesting relay network has been considered in many studies. Such as, [5] investigated the full-duplex energy harvesting relay network with the simultaneous energy harvesting and information transmission. Moreover, the development of cooperative protocols for energy harvesting relay network is deeply studied in [6-7]. Furthermore, [8-9] proposed a "harvest-then-transmit" protocol for a multi-user relay network. In all papers above, the energy source of the whole network is only the source or the access point. In the trends to improving the energy harvesting and information transmission processes in the wireless relay network, some researchers proposed the idea of deploying dedicated power beacon node (PB). In the wireless relay network with using the PB node, the destination (D) can harvest wireless energy 
not only from the source (S) node but also from the deployed PB node [10-11]. For this new model, the question about system performance is still open and is necessary to investigate. However, there are not many works concentrate on the Rician fading channel yet, and the outage performance analysis of such channels is fundamental to practice. The remaining gap can fill by this work.

In this work, we propose and demonstrate the system performance of the EH DF HF relaying network in the case of maximizing and non-maximizing ergodic capacity. For details on this analysis, the energy, and information are transferred from the source to the relay nodes by the power splitting protocol, and all channels are considered as the Rician fading channels. The main contributions of the paper are summarized as follows:

1) The system model of the half-duplex relaying network over the Rician fading channels is proposed with the PS protocol.

2) The mathematical expressions of the ergodic capacity in cases maximize, and non-maximize of the system model are derived.

3) The influence of the main parameters on the system performance is demonstrated entirely by the Monte Carlo simulation.

The structure of this paper is proposed as follows. Sections II presents the system model of the relaying network. Sections III derive the analytical expression of ergodic capacity in cases maximize and non-maximize of the model system. Section IV provides the numerical results and some discussions. Finally, Section V concludes the paper.

\section{SYSTEM MODEL}

In this paper, the system model is the energy harvesting half-duplex (HF) decode-and-forward (DF) power splitting (PS) protocol relaying network, as shown in Figure 1. In this model, the information is transferred from the source (S) to the destination (D), through energy constrained intermediate relay (R). The energy harvesting and information processing of the system model with PS protocol are proposed in Figure 2. In this scheme, $\mathrm{T}$ is the block time in which the source fully transmit the information data to the destination. In the first interval time $(T / 2)$, the relay harvests energy $(\rho T)$ and receives information $((1-\rho) T)$ from the source signal, where $\rho$ is the power splitting factor $\rho \in(0,1)$. In the remaining half-time $T / 2$, the relay node transfers information to the destination node. All the fading channels from $\mathrm{S}$ to $\mathrm{R}$ and $\mathrm{R}$ to $\mathrm{D}$ are proposed as the Rician fading channels. More details of the analytical mathematical model of the ergodic capacity of the system model is presented and analyzed in the following sections [12-16], [18].

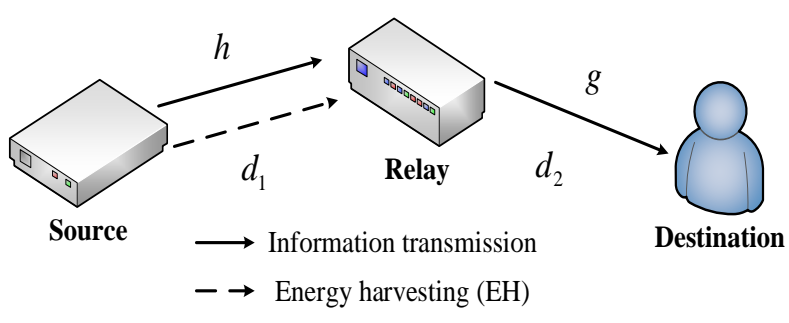

Figure 1. System model

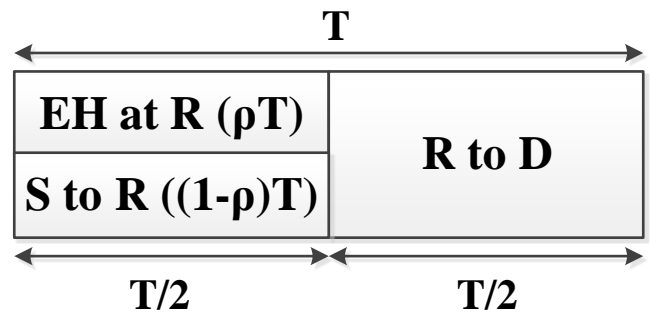

Figure 2. The power splitting protocol

\section{THE SYSTEM PERFORMANCE}

In this section, we analyzed and demonstrated the system performance analysis of the model system in the power splitting protocol [12-14]. In the first transmission phase based on the above system model, the received signal at the relay can be given by (1):

$$
y_{r}=\frac{1}{\sqrt{d_{1}^{m}}} \sqrt{(1-\rho)} h x_{s}+n_{r}
$$

where $h$ is the source to relay channel gain, $\mathrm{d}_{1}$ is the source to relay distance, and $m$ is the path loss exponent. 
Here, $x_{s}$ is the transmitted signal at the source, $\mathrm{n}_{\mathrm{r}}$ is the additive white Gaussian noise (AWGN) with variance $\mathrm{N}_{0}$ and $0<\rho<1$ is power splitting ratio at the relay. Moreover, $\mathrm{E}\left\{\left|x_{s}\right|^{2}\right\}=P_{s}, \mathrm{E}\{\bullet\}$ : expectation operator, and $\mathrm{P}_{\mathrm{s}}$ is average transmit power at the source.

$$
P_{r}=\frac{E_{h}}{d_{1}^{m}(T / 2)}=\frac{\eta \rho P_{s}|h|^{2}(T / 2)}{d_{1}^{m}(T / 2)}=\frac{\eta \rho P_{s}|h|^{2}}{d_{1}^{m}}
$$

In (2), $0<\eta \leq 1$ is an energy conversion efficiency coefficient.

In this system model, we proposed that the model is working in the decode-and-forward mode. Then the signal to noise ratio (SNR) at the information processing phase of the relay can be calculated by:

$$
S N R_{s, r}=\frac{(1-\rho) P_{s}|h|^{2}}{d_{1}^{m} N_{0}}
$$

From the equation (3), the capacity of S-R link can be calculated as:

$$
C_{s, r}=\frac{1}{2} \log _{2}\left(1+S N R_{s, r}\right)=\frac{1}{2} \log _{2}\left[1+\frac{(1-\rho) P_{s}|h|^{2}}{d_{1}^{m} N_{0}}\right]
$$

In the same way, the received signal at the destination in the second phase can be expressed as:

$$
y_{d}=\frac{1}{\sqrt{d_{2}^{m}}} g x_{r}+n_{d}
$$

Where $g$ is the source to relay channel gain, $\mathrm{d} 2$ is the relay to destination distance, and $\mathrm{E}\left\{\left|x_{r}\right|^{2}\right\}=P_{r}$ By combining with the equation (2), the SNR of the destination can be expressed as:

$$
S N R_{r, d}=\frac{P_{r}|g|^{2}}{d_{2}^{m} N_{0}}=\frac{\eta \rho P_{s}|h|^{2}|g|^{2}}{d_{1}^{m} d_{2}^{m} N_{0}}
$$

The capacity of R-D link can be calculated as:

$$
C_{r, d}=\frac{1}{2} \log _{2}\left[1+\frac{\eta \rho P_{s}|h|^{2}|g|^{2}}{d_{1}^{m} d_{2}^{m} N_{0}}\right]
$$

In this analysis, we consider $\mathrm{h}, \mathrm{g}$ gain factor as Rician fading channel, and $\mathrm{h}, \mathrm{g}$ have a random distribution. From that, the probability density function (PDF) of a random variable (RV) $\varphi_{i}$ where $\mathrm{i}=1,2$ is formulated as in [14].

Where $\varphi_{1}=|h|^{2}, \varphi_{2}=|g|^{2}$

$$
f_{\varphi_{i}}(x)=a \sum_{l=0}^{\infty} \frac{(b K)^{l}}{(l !)^{2}} x^{l} e^{-b x}
$$


Where we denote $a=\frac{(K+1) e^{-K}}{\lambda_{i}}, b=\frac{K+1}{\lambda_{i}}$

In (8), $\lambda_{i}$ is the mean value of $\operatorname{RV} \varphi_{i}$ which $i=1,2$ respectively and $\lambda_{1}=\frac{\mathrm{E}\left\{|h|^{2}\right\}}{d_{1}^{m}}, \lambda_{2}=\frac{\mathrm{E}\left\{|g|^{2}\right\}}{d_{2}^{m}}$. Moreover, $\mathrm{K}$ is the Rician K-factor defined as the ratio of the power of the line-of-sight (LOS) component to the scattered components and $I_{0}(\bullet)$ is the zero-th order modified Bessel function of the first kind.

Here, the cumulative density function(CDF) of $\mathrm{RV} \varphi_{i}$ where $\mathrm{i}=1,2$ can be computed as in [14]. We assume that $\lambda_{1}=\lambda_{2}$, then we have:

$$
F_{\varphi_{i}}(\varsigma)=\int_{0}^{\varsigma} f_{\varphi_{i}}(x) d x=1-\frac{a}{b} \sum_{l=0}^{\infty} \sum_{n=0}^{l} \frac{K^{l} b^{n}}{l ! n !} \varsigma^{n} e^{-b \varsigma}
$$

\subsection{Maximize capacity Case}

For decode-and-forward relaying the overall Ergodic capacity and SNR from source to destination can be written as follows:

$$
\begin{aligned}
& C_{D F}=\min \left(C_{s, r}, C_{r, d}\right)=\min \left[\frac{1}{2} \log _{2}\left(1+\frac{(1-\rho) P_{s}|h|^{2}}{d_{1}^{m} N_{0}}\right), \frac{1}{2} \log _{2}\left(1+\frac{\eta \rho P_{s} \varphi_{1} \varphi_{2}}{d_{1}^{m} d_{2}^{m} N_{0}}\right)\right] \\
& S N R^{D F}=\min \left(S N R_{s, r}, S N R_{r, d}\right)=\min \left(\frac{(1-\rho) P_{s}|h|^{2}}{d_{1}^{m} N_{0}}, \frac{\eta \rho P_{s}|h|^{2}|g|^{2}}{d_{1}^{m} d_{2}^{m} N_{0}}\right)
\end{aligned}
$$

In equations (9) and (10), we can obtain the following value of $\rho$ maximizes the $C_{D F}$ and the same for $S N R^{D F}$ as the following:

$$
\rho_{D F}^{*}=\frac{1}{\frac{\eta \varphi_{2}}{d_{2}^{m}}+1}
$$

Proof: See the Appendix A.

\subsection{Non-maximize capacity case}

In the case non-maximize capacity, we can calculate the ergodic capacity as:

$$
\begin{aligned}
& C_{s, r}=\frac{1}{\ln 2} \int_{0}^{\infty} \frac{1-F_{S N R_{s, r}}(\gamma)}{1+\gamma} d \gamma \\
& C_{r, d}=\frac{1}{\ln 2} \int_{0}^{\infty} \frac{1-F_{S N R_{r, d}}(\gamma)}{1+\gamma} d \gamma
\end{aligned}
$$

From these equations, we have:

$$
\begin{aligned}
& F_{S N R_{s, r}}(\gamma)=\operatorname{Pr}\left(S N R_{s, r}<\gamma\right)=\operatorname{Pr}\left(\frac{(1-\rho) P_{s}|h|^{2}}{d_{1}^{m} N_{0}}<\gamma\right) \\
& =\operatorname{Pr}\left(\varphi_{1}<\frac{\gamma d_{1}^{m}}{(1-\rho) \gamma_{0}}\right)=F_{\varphi_{1}}\left(\frac{\gamma d_{1}^{m}}{(1-\rho) \gamma_{0}}\right)
\end{aligned}
$$


From the equation (8), we have:

$$
\begin{aligned}
& F_{\varphi_{1}}\left(\frac{\gamma d_{1}^{m}}{(1-\rho) \gamma_{0}}\right)=1-\frac{a}{b} \sum_{l=0}^{\infty} \sum_{n=0}^{l} \frac{K^{l} b^{n}}{l ! n !}\left[\frac{\gamma d_{1}^{m}}{(1-\rho) \gamma_{0}}\right]^{n} e^{-\frac{b \gamma d_{1}^{m}}{(1-\rho) \gamma_{0}}} \\
& F_{S N R_{r, d}}(\gamma)=\operatorname{Pr}\left(S N R_{r, d}<\gamma\right)=\operatorname{Pr}\left(\frac{\eta \rho P_{s}|h|^{2}|g|^{2}}{d_{1}^{m} d_{2}^{m} N_{0}}<\gamma\right)=\operatorname{Pr}\left(\varphi_{1}<\frac{\gamma d_{1}^{m} d_{2}^{m}}{\eta \rho \gamma_{0} \varphi_{2}}\right) \\
& F_{S N R_{r, d}}(\gamma)=\int_{0}^{\infty} F_{\varphi_{1}}\left(\frac{\gamma d_{1}^{m} d_{2}^{m}}{\eta \rho \gamma_{0} \varphi_{2}} \mid \varphi_{2}\right) f_{\varphi_{2}}\left(\varphi_{2}\right) d \varphi_{2}
\end{aligned}
$$

Similarity,

$$
\begin{aligned}
& F_{S N R_{r, d}}(\gamma)=1-a^{2} \sum_{l=0}^{\infty} \sum_{k=0}^{\infty} \sum_{n=0}^{l} \frac{K^{l+k} b^{n+k-1}}{l ! n !(k !)^{2}} \int_{0}^{\infty}\left(\frac{\gamma d_{1}^{m} d_{2}^{m}}{\eta \rho \gamma_{0} \varphi_{2}}\right)^{n} e^{-\frac{b \gamma d_{1}^{m} \gamma_{2}^{m}}{\eta \gamma_{0} \varphi_{2}}} \varphi_{2}^{k} e^{-b \varphi_{2}} d \varphi_{2} \\
& F_{S N R_{r, d}}(\gamma)=1-a^{2} \sum_{l=0}^{\infty} \sum_{k=0}^{\infty} \sum_{n=0}^{l} \frac{K^{l+k} b^{n+k-1}}{l ! n !(k !)^{2}}\left(\frac{\gamma d_{1}^{m} d_{2}^{m}}{\eta \rho \gamma_{0}}\right)^{n} \int_{0}^{\infty} \varphi_{2}^{k-n} e^{-\frac{b \gamma d_{1}^{m} d_{2}^{m}}{\eta \rho \gamma_{0} \varphi_{2}}} e^{-b \varphi_{2}} d \varphi_{2}
\end{aligned}
$$

Apply [3.471,9] of table of integral [17], we have:

$$
F_{S N R_{r, d}}(\gamma)=1-2 a^{2} \sum_{l=0}^{\infty} \sum_{k=0}^{\infty} \sum_{n=0}^{l} \frac{K^{l+k} b^{n+k-1}}{l ! n !(k !)^{2}}\left(\frac{\gamma d_{1}^{m} d_{2}^{m}}{\eta \rho \gamma_{0}}\right)^{\frac{k+n+1}{2}} \times K_{k-n+1}\left(2 b \sqrt{\frac{\gamma d_{1}^{m} d_{2}^{m}}{\eta \rho \gamma_{0}}}\right)
$$

Replace (15) into (12), (20) into (13), finally we have:

$$
\begin{aligned}
& C_{s, r}=\frac{a}{\ln 2} \int_{0}^{\infty} \frac{1}{(1+\gamma)} \sum_{l=0}^{\infty} \sum_{n=0}^{l} \frac{K^{l} b^{n-1}}{l ! n !}\left[\frac{\gamma d_{1}^{m}}{(1-\rho) \gamma_{0}}\right]^{n} e^{-\frac{b \gamma d_{1}^{m}}{(1-\rho) \gamma_{0}}} d \gamma \\
& C_{r, d}=\frac{2 a^{2}}{\ln 2} \int_{0}^{\infty} \frac{1}{(1+\gamma)} \sum_{l=0}^{\infty} \sum_{k=0}^{\infty} \sum_{n=0}^{l} \frac{K^{l+k} b^{n+k-1}}{l ! n !(k !)^{2}}\left(\frac{\gamma d_{1}^{m} d_{2}^{m}}{\eta \rho \gamma_{0}}\right)^{\frac{k+n+1}{2}} \times K_{k-n+1}\left(2 b \sqrt{\frac{\gamma d_{1}^{m} d_{2}^{m}}{\eta \rho \gamma_{0}}}\right) d \gamma \\
& C_{D F}=\min \left(C_{s, r}, C_{r, d}\right)
\end{aligned}
$$

\section{NUMERICAL RESULTS AND DISCUSSION}

In this section, the Monte Carlo simulation is used for validating the analytical expression in the above section. The system performance in term of ergodic capacity is analyzed and demonstrated in connection with $\eta, d_{1}=d_{2}, P_{s} / N_{0}$ and $K$. We consider a network with one source, one relay, and one destination, where source-relay and relay-destination distances are both normalized to unit value.

The effect of $\mathrm{P}_{\mathrm{s}} / \mathrm{N}_{0}$ for the power splitting protocol on the ergodic capacity of the proposed relay network system is presented in Figure3. Figure 3 plots the ergodic capacity of the system model for maximize case and for a non-maximize case with the power splitting factor of 0.2 and 0.5 . In Figure 3 , we set the system parameters as $\mathrm{d}_{1}=0.65, \mathrm{~d}_{2}=0.85, \eta=0.8$ and $\mathrm{K}=2$. From the results, the analytical and the simulation results match for all possible values $\mathrm{P}_{\mathrm{s}} / \mathrm{N}_{0}$. Figure 4 shows that the ergodic capacity has a considerable increase while $\mathrm{P}_{\mathrm{s}} / \mathrm{N}_{0}$ increases from 0 to 30 . Moreover, the ergodic capacity in maximize case in better than others cases.

Furthermore, the ergodic capacity of the proposed system versus $d_{1}=d_{2}$ is illustrated in the Figure 4 with $\mathrm{P}_{\mathrm{S}} / \mathrm{N}_{0}=20$ and $\mathrm{K}=2$. From the results, we show that the ergodic capacity decreases significantly with 
increasing the distances $d_{1}=d_{2}$ from 0.5 to 1.5 . It can be prove by the opinion that the strength of the RF signal decreases in the direction of increasing transmission distance between $\mathrm{S}, \mathrm{R}$ and $\mathrm{D}$. All the analytical and simulation results are agree well with each other. In the same way, the influence of $\mathrm{K}$ on the ergodic capacity of the system model in both maximize and non-maximize cases is proposed in Figure 5. In Figure 5, we set $P_{s} / N_{0}=10, \rho=0.3$ and $\eta=0.8$. From the results, the ergodic capacity in all cases has slight increase in the increasing trend of the $\mathrm{K}$ from 0 to 4 . Finally, Figure 6 illustrates the effect of $\eta$ versus ergodic capacity with the main parameters of $P_{s} / N_{0}=20, K=2$, and $\eta=0.8$. The ergodic capacity increases remarkably while $\eta$ increases from 0 to 1 . The results show that all simulation and analytical results are matched well with each other. Moreover, the ergodic capacity in case maximize is better than the remaining cases.

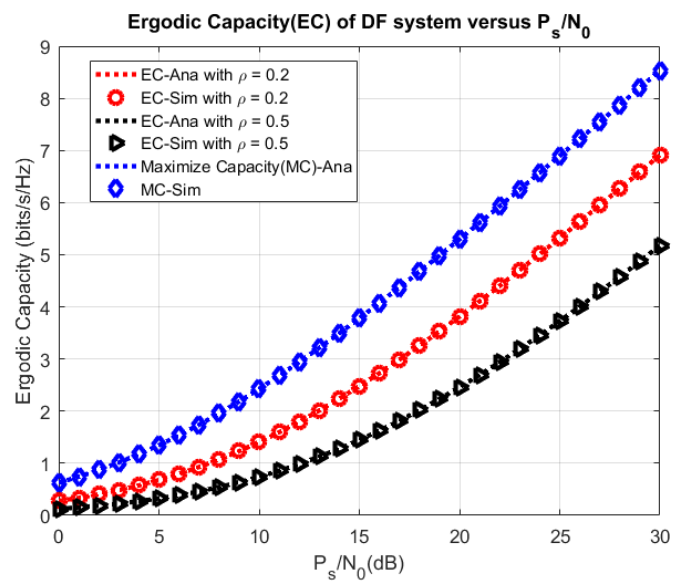

Figure 3. Ergodic capacity versus $\mathrm{P}_{\mathrm{s}} / \mathrm{N}_{0}$

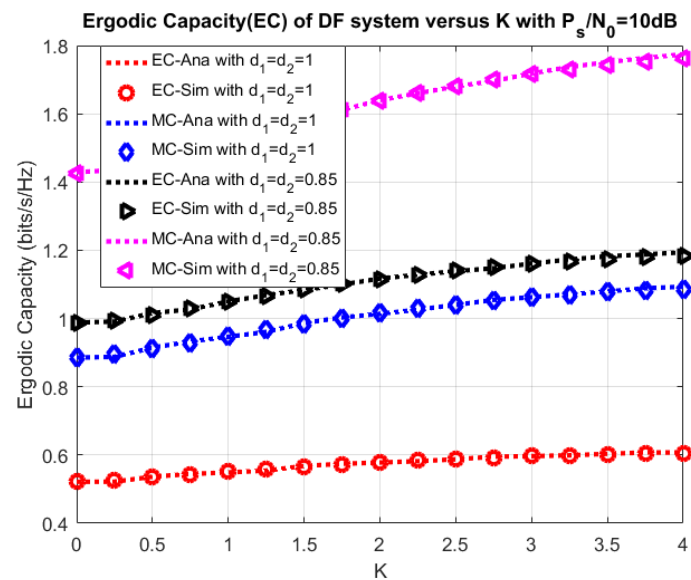

Figure 5. Ergodic capacity versus K

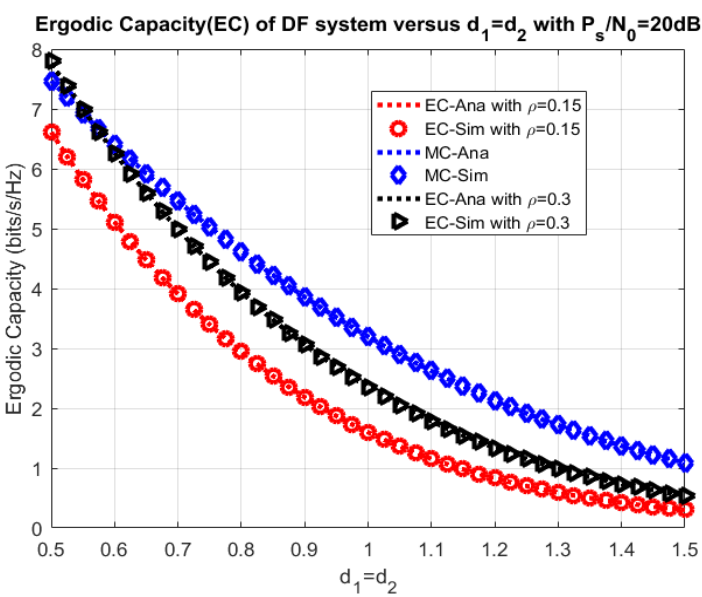

Figure 4. Ergodic capacity versus $\mathrm{d}_{1}=\mathrm{d}_{2}$

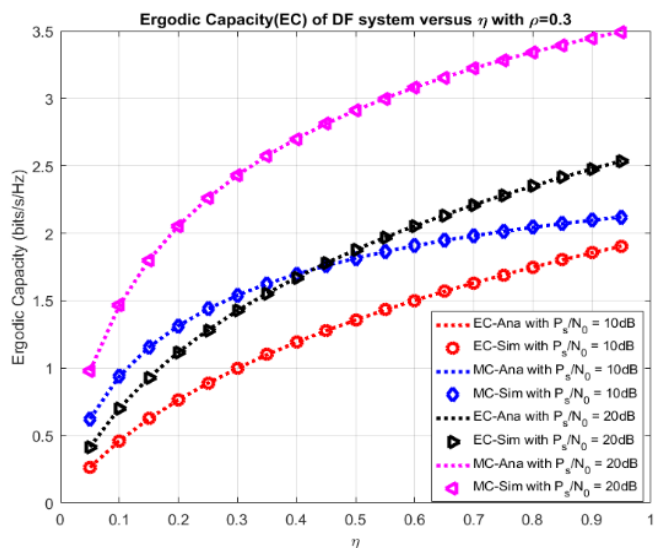

Figure 6. Ergodic capacity versus $\eta$

\section{CONCLUSION}

In this paper, we investigate the system performance of the EH half-duplex DF relaying network over the Rician fading channel in power splitting protocol. For performance analysis system, we derive the analytical expressions of ergodic capacity for both maximize and non-maximize cases. The Monte Carlo simulation is used for validating the analytical method. The results show that the analytical mathematical and simulated results match for all possible parameter values for both schemes and the maximize case provides the better results in comparison with the non-maximize case of the ergodic capacity. The results could provide the prospective recommendation for the communication network in the near future. 


\section{APPENDIX A.}

We can see that $C_{D F}$ is maximized when all of its argument becomes equal. Hence, the value of $\rho_{D F}^{*}$ can be obtained by equating $\mathrm{C}_{\mathrm{s}, \mathrm{r}}$ to $\mathrm{C}_{\mathrm{r}, \mathrm{d}}$. Replace (12) into (11) we have:

$$
S N R^{D F}=\frac{\eta P_{s} \varphi_{1} \varphi_{2}}{\left(\frac{\eta \varphi_{2}}{d_{2}^{m}}+1\right) d_{1}^{m} d_{2}^{m} N_{0}}=\frac{\eta \gamma_{0} \varphi_{1} \varphi_{2}}{\eta \varphi_{2} d_{1}^{m}+d_{1}^{m} d_{2}^{m}}
$$

Where we denote $\gamma_{0}=\frac{P_{s}}{N_{0}}$.

Then the Ergodic capacity can be calculated as:

$$
\begin{aligned}
& C_{D F}=\int_{0}^{\infty} f_{S N R^{D F}}(\gamma) \log _{2}(1+\gamma) d \gamma=\frac{1}{\ln 2} \int_{0}^{\infty} \frac{1-F_{S N R^{D F}}(\gamma)}{1+\gamma} d \gamma \\
& F_{S N R^{D F}}(\gamma)=\operatorname{Pr}\left(S N R^{D F}<\gamma\right)=\operatorname{Pr}\left(\frac{\eta \gamma_{0} \varphi_{1} \varphi_{2}}{\eta \varphi_{2} d_{1}^{m}+d_{1}^{m} d_{2}^{m}}<\gamma\right) \\
& =\operatorname{Pr}\left[\varphi_{1}<\frac{\gamma\left(\eta \varphi_{2} d_{1}^{m}+d_{1}^{m} d_{2}^{m}\right)}{\eta \gamma_{0} \varphi_{2}}\right]=\int_{0}^{\infty} F_{\varphi_{1}}\left[\frac{\gamma\left(\eta \varphi_{2} d_{1}^{m}+d_{1}^{m} d_{2}^{m}\right)}{\eta \gamma_{0} \varphi_{2}} \mid \varphi_{2}\right] f_{\varphi_{2}}\left(\varphi_{2}\right) d \varphi_{2}
\end{aligned}
$$

Where $\gamma$ is a threshold of the system.

Furthermore, from the equations (3), (4) we have:

$$
\begin{aligned}
& F_{S N R^{D F}}(\gamma)=1-\int_{0}^{\infty} \frac{a^{2}}{b} \sum_{l=0}^{\infty} \sum_{n=0}^{l} \frac{K^{l} b^{n}}{l ! n !}\left[\frac{\gamma\left(\eta \varphi_{2} d_{1}^{m}+d_{1}^{m} d_{2}^{m}\right)}{\eta \gamma_{0} \varphi_{2}}\right]^{n} \\
& \exp \left[-\frac{b \gamma\left(\eta \varphi_{2} d_{1}^{m}+d_{1}^{m} d_{2}^{m}\right)}{\eta \gamma_{0} \varphi_{2}}\right] \sum_{l=0}^{\infty} \frac{(b K)^{l}}{(l !)^{2}} \varphi_{2}^{l} e^{-b \varphi_{2}} d \varphi_{2} \\
& F_{S N R^{D F}}(\gamma)=1-\int_{0}^{\infty} a^{2} \sum_{l=0}^{\infty} \sum_{k=0}^{\infty} \sum_{n=0}^{l} \frac{K^{l+k} b^{n+k-1}}{l ! n !(k !)^{2}}\left(\frac{\gamma d_{1}^{m}}{\gamma_{0}}\right)^{n}\left[1+\frac{d_{2}^{m}}{\eta \varphi_{2}}\right]^{n} \\
& \exp \left[-\frac{b \gamma\left(\eta \varphi_{2} d_{1}^{m}+d_{1}^{m} d_{2}^{m}\right)}{\eta \gamma_{0} \varphi_{2}}\right] \varphi_{2}^{k} e^{-b \varphi_{2}} d \varphi_{2}
\end{aligned}
$$

In this analysis, by applying the equation $(x+y)^{n}=\sum_{t=0}^{n}\left(\begin{array}{l}n \\ t\end{array}\right) x^{n-t} y^{t}$ to (20), the outage probability can be demonstrated as follow:

$$
\begin{aligned}
& F_{S N R^{D F}}(\gamma)=1-\int_{0}^{\infty} a^{2} \sum_{l=0}^{\infty} \sum_{k=0}^{\infty} \sum_{n=0}^{l} \sum_{t=0}^{n} \frac{K^{l+k} b^{n+k-1}}{l ! n !(k !)^{2}}\left(\frac{\gamma d_{1}^{m}}{\gamma_{0}}\right)^{n}\left(\begin{array}{l}
n \\
t
\end{array}\right)\left(\frac{d_{2}^{m}}{\eta \varphi_{2}}\right)^{t} e^{-\frac{b \gamma d_{1}^{m}}{\gamma_{0}}} e^{-\frac{b \gamma d_{1}^{m} d_{2}^{m}}{\eta \gamma_{0} \varphi_{2}}} \varphi_{2}^{k} e^{-b \varphi_{2}} d \varphi_{2} \\
& F_{S N R^{D F}}(\gamma)=1-a^{2} e^{-\frac{b \gamma d_{1}^{m}}{\gamma_{0}}} \sum_{l=0}^{\infty} \sum_{k=0}^{\infty} \sum_{n=0}^{l} \sum_{t=0}^{n} \frac{K^{l+k} b^{n+k-1}}{l ! t !(n-t) !(k !)^{2}}\left(\frac{\gamma d_{1}^{m}}{\gamma_{0}}\right)^{n}\left(\frac{d_{2}^{m}}{\eta}\right)^{t} \int_{0}^{\infty} \varphi_{2}^{k-t} e^{-\frac{b \gamma d_{1}^{m} d_{2}^{m}}{\eta \gamma_{0} \varphi_{2}}} e^{-b \varphi_{2}} d \varphi_{2}
\end{aligned}
$$

Apply the equation [3.471,9] of table of integral [], we have: 


$$
\begin{aligned}
& F_{S N R^{D F}}(\gamma)=1-2 a^{2} e^{-\frac{b \gamma d_{1}^{m}}{\gamma_{0}}} \sum_{l=0}^{\infty} \sum_{k=0}^{\infty} \sum_{n=0}^{l} \sum_{t=0}^{n} \frac{K^{l+k} b^{n+k-1}}{l ! t !(n-t) !(k !)^{2}}\left(\frac{\gamma d_{1}^{m}}{\gamma_{0}}\right)^{n}\left(\frac{d_{2}^{m}}{\eta}\right)^{t}\left(\frac{\gamma d_{1}^{m} d_{2}^{m}}{\eta \gamma_{0}}\right)^{\frac{k-t+1}{2}} \\
& \times K_{k-t+1}\left(2 b \sqrt{\frac{\gamma d_{1}^{m} d_{2}^{m}}{\eta \gamma_{0}}}\right) \\
& F_{S N R^{D F}}(\gamma)=1-2 a^{2} e^{-\frac{b \gamma d_{1}^{m}}{\gamma_{0}}} \sum_{l=0}^{\infty} \sum_{k=0}^{\infty} \sum_{n=0}^{l} \sum_{t=0}^{n} \frac{K^{l+k} b^{n+k-1}}{l ! t !(n-t) !(k !)^{2}}\left(\frac{\gamma d_{1}^{m}}{\gamma_{0}}\right)^{\frac{2 n+k-t+1}{2}} \\
& \left(\frac{d_{2}^{m}}{\eta}\right)^{\frac{k+t+1}{2}} \times K_{k-t+1}\left(2 b \sqrt{\frac{\gamma d_{1}^{m} d_{2}^{m}}{\eta \gamma_{0}}}\right)
\end{aligned}
$$

Where $K_{v}(\bullet)$ is the modifed Bessel function of the second kind and $\mathrm{v}^{\text {th }}$ order.

Finally, we have:

$$
\begin{aligned}
& C_{D F}=\frac{2 a^{2}}{\ln 2} \sum_{l=0}^{\infty} \sum_{k=0}^{\infty} \sum_{n=0}^{l} \sum_{t=0}^{n} \frac{K^{l+k} b^{n+k-1}}{l ! t !(n-t) !(k !)^{2}}\left(\frac{d_{2}^{m}}{\eta}\right)^{\frac{k+t+1}{2}} \\
& \int_{0}^{\infty} \frac{\left(\frac{\gamma d_{1}^{m}}{\gamma_{0}}\right)^{\frac{2 n+k-t+1}{2}} \times K_{k-t+1}\left(2 b \sqrt{\frac{\gamma d_{1}^{m} d_{2}^{m}}{\eta \gamma_{0}}}\right) e^{-\frac{b \gamma d_{1}^{m}}{\gamma_{0}}}}{1+\gamma} d \gamma
\end{aligned}
$$

End of Proof.

\section{REFERENCES}

[1] Bi, S., Ho, C. K., \& Zhang, R. (2015, 04). Wireless powered communication: Opportunities and challenges. IEEE Communications Magazine, 53(4), 117-125. doi:10.1109/mcom.2015.7081084.

[2] Niyato, D., Kim, D. I., Maso, M., \& Han, Z. (2017). Wireless Powered Communication Networks: Research Directions and Technological Approaches. IEEE Wireless Communications, 2-11. doi:10.1109/mwc.2017.1600116.

[3] Nasir, A. A., Zhou, X., Durrani, S., \& Kennedy, R. A. (2013, 07). Relaying Protocols for Wireless Energy Harvesting and Information Processing. IEEE Transactions on Wireless Communications, 12(7), 3622-3636. doi:10.1109/twc.2013.062413.122042.

[4] Baidas, M. W., \& Alsusa, E. A. (2016, 02). Power allocation, relay selection and energy cooperation strategies in energy harvesting cooperative wireless networks. Wireless Communications and Mobile Computing, 16(14), 20652082. doi: $10.1002 / \mathrm{wcm} .2668$.

[5] Krikidis, I. (2014, 03). Simultaneous Information and Energy Transfer in Large-Scale Networks with/without Relaying. IEEE Transactions on Communications, 62(3), 900-912. doi:10.1109/tcomm.2014.020914.130825.

[6] Ding, Z., Krikidis, I., Sharif, B., \& Poor, H. V. (2014, 08). Wireless Information and Power Transfer in Cooperative Networks With Spatially Random Relays. IEEE Transactions on Wireless Communications, 13(8), 4440-4453. doi:10.1109/twc.2014.2314114.

[7] Mheich, Z., \& Savin, V. (2017, 03). Cooperative communication protocols with energy harvesting relays. 2017 Wireless Days. doi:10.1109/wd.2017.7918116.

[8] Huang, C., Zhang, R., \& Cui, S. (2013, 08). Throughput Maximization for the Gaussian Relay Channel with Energy Harvesting Constraints. IEEE Journal on Selected Areas in Communications, 31(8), 1469-1479. doi:10.1109/jsac.2013.130811.

[9] Ahmed, I., Ikhlef, A., Schober, R., \& Mallik, R. K. (2013, 04). Joint Power Allocation and Relay Selection in Energy Harvesting AF Relay Systems. IEEE Wireless Communications Letters, 2(2), 239-242. doi:10.1109/wcl.2013.012513.130007.

[10] Zeng, Y., Chen, H., \& Zhang, R. (2016, 05). Bidirectional Wireless Information and Power Transfer With a Helping Relay. IEEE Communications Letters, 20(5), 862-865. doi:10.1109/lcomm.2016.2549515.

[11] Gurakan, B., Ozel, O., Yang, J., \& Ulukus, S. (2013, 06). Energy cooperation in energy harvesting two-way communications. 2013 IEEE International Conference on Communications (ICC). doi:10.1109/icc.2013.6655023.

[12] Chaudhry, M., \& Zubair, S. (2002, 10). Extended incomplete gamma functions with applications. Journal of Mathematical Analysis and Applications, 274(2), 725-745. doi:10.1016/s0022-247x(02)00354-2 . 
[13] J., J. E. (1979, 08). The H-Function with Applications in Statistics and Other Disciplines. Technometrics, 21(3), 392-393. doi:10.1080/00401706.1979.10489802.

[14] Table of Integrals, Series, and Products. (2015). doi:10.1016/c2010-0-64839-5.

[15] Tran Hoang Quang Minh. "Hybrid Time-Power Switching Protocol of Energy Harvesting Bidirectional Relaying Network: Throughput and Ergodic Capacity Analysis." TELKOMNIKA (Telecommunication Computing Electronics and Control), 16, no. 5 (10, 2018): 189. http://dx.doi.org/10.12928/telkomnika.v16i5.9118.

[16] Tin, Phu Tran, Tran Hoang Quang Minh, Tan N. Nguyen, and Miroslav Voznak. "System Performance Analysis of Half-Duplex Relay Network over Rician Fading Channel." TELKOMNIKA (Telecommunication Computing Electronics and Control). 16, no. 1 (02, 2018): 189. doi:10.12928/telkomnika.v16i1.7491.

[17] Rashid, Tarique, Sunil Kumar, Akshay Verma, Prateek Raj Gautam, and Arvind Kumar. "Pm-EEMRP: Postural Movement Based Energy Efficient Multi-hop Routing Protocol for Intra Wireless Body Sensor Network (IntraWBSN)." TELKOMNIKA Telecommunication Computing Electronics and Control), 16, no. 1 (02, 2018): 166. doi:10.12928/telkomnika.v16i1.7318.

[18] A. F. Morabito, "Power Synthesis of Mask-Constrained Shaped Beams Through Maximally-Sparse Planar Arrays," TELKOMNIKA (Telecommunication Computing Electronics and Control), vol. 14, n. 4, pp. 1217-1219, 2016. 